\title{
PySPH: A Python Framework for Smoothed Particle Hydrodynamics
}

\author{
Prabhu Ramachandran ${ }^{\ddagger *}$, Chandrashekhar Kaushik
}

\begin{abstract}
PySPH}]$ is a Python-based open source parallel framework for Smoothed Particle Hydrodynamics (SPH) simulations. It is distributed under a BSD license. The performance critical parts are implemented in [Cython]. The framework provides a load balanced, parallel execution of solvers. It is designed to be easy to extend. In this paper we describe the architecture of $\mathrm{PySPH}$ and how it can be used.

At it's core PySPH provides a particle kernel, an SPH kernel and a solver framework. Serial and parallel versions of solvers for some standard problems are also provided. The parallel solver uses [mpi4py]. We employ a simple but elegant automatic load balancing strategy for the parallelization. Currently, we are able to perform free surface simulations and some gas dynamics simulations. PySPH is still a work in progress and we will discuss our future plans for the project.
\end{abstract}

Index Terms - parallel, Cython, fluid dynamics, simulation

\section{Introduction}

\section{SPH Primer}

Smoothed Particle Hydrodynamics (SPH) is a computational simulation technique. It was developed to simulate astral phenomena by [Gingold77] and [Lucy77] in 1977. Since then, it has been used in numerous other fields including fluid-dynamics, gas-dynamics and solid mechanics.

The central idea behind SPH is the use of integral interpolants. Consider a function $f(r)$. It can be represented by the equation

$$
f(r)=\int f\left(r^{\prime}\right) \delta\left(r-r^{\prime}\right) d r^{\prime}
$$

Replacing the delta distribution with an approximate delta function, $W$, gives us:

$$
f(r)=\int f\left(r^{\prime}\right) W\left(r-r^{\prime}, h\right) d r^{\prime}
$$

The above equation estimates the value of function $f$ at a point $r$ in space using the weighted values of $f$ at points near it. The weight decreases as the distance between $r$ and $r^{\prime}$ increase. $h$ in the above equation represents the particle interaction radius. The support of the kernel $W$ is some small multiple of $h$. Outside the support, the value of $W$ is set to zero. Compact support is computationally advantageous since it allows us to avoid an $N^{2}$ interaction among particles.

* Corresponding author: prabhu@aero.iitb.ac.in + IIT Bombay

Copyright $\odot 2010$ Prabhu Ramachandran et al. This is an open-access article distributed under the terms of the Creative Commons Attribution License, which permits unrestricted use, distribution, and reproduction in any medium, provided the original author and source are credited.
The above equation can be written in summation form as

$$
f\left(r_{i}\right)=\sum_{j} f\left(r_{j}\right) \frac{m_{j}}{\rho_{j}} W\left(r_{i}-r_{j}, h\right)
$$

The above equation forms the core of all SPH calculations. The index $j$ loops over all neighboring particles. $m_{j}$ is the mass of a particle and $\rho_{j}$ is the density of the particle. The term

$$
\frac{m_{j}}{\rho_{j}}
$$

can be thought of as representing a volume element [Morris96]. Gradients and divergence encountered in the equations representing fluid motion are represented using similar summations. SPH finds widespread use in many domains. [Monaghan05] and [Morris97] give extensive details about the SPH method.

\section{Related Work}

Despite the age of SPH and its applicability to many domains, there does not seem to be much effort in developing a unified framework for SPH. [SPHysics] is a FORTRAN-based open source package for performing SPH. It's primary objective is to model free-surface flows. From the provided documentation we feel that it is not easy to set up simulations in this package. [SPH2000] is another parallel framework for SPH written in $\mathrm{C}++$. This code however does not seem to be in active development currently. Moreover, they show exactly one example simulation with their code. Neither package has a publicly accessible source code repository. Therefore, an open source package that is easy to experiment with and extend will be a useful contribution to the community, especially when combined with the flexibility of Python [Oliphant07].

PySPH [PySPH] was created to address this need. It is an open source, parallel, framework for Smoothed Particle Hydrodynamics (SPH) implemented in Python.

\section{Choice of implementation language}

We use a combination of Python and [Cython] to implement the framework. Python is a high-level, object-oriented, interpreted programming language which is easy to learn. Python code is also very readable. There are numerous packages (both scientific and otherwise) that can be used to enhance the productivity of applications. A Python-based SPH implementation can take advantage of these packages, which could enhance it in various aspects, from providing plotting facilities (2D and 3D), to generating GUI's, to running SPH simulations from the web, to parallelization. All these features can also be accessed through an interactive 
interpreter. [Oliphant07] discusses how Python can be used for scientific computing.

Python, however, is an interpreted language. Thus, computeintensive tasks implemented in pure Python will be prohibitively slow. To overcome this, we delegate all performance-critical tasks to a language called Cython [Cython]. Cython makes writing $\mathrm{C}$ extensions for Python nearly as simple as writing Python code itself. A Cython module is compiled by a compiler into a $\mathrm{C}$ extension module. When the $\mathrm{C}$ code is compiled, it becomes a module that may be imported from Python. Most of Python's features are available in Cython. Thus, by delegating all performance-critical components to Cython, we are able to overcome the performance hit due to the interpreted nature of Python and still use all of Python's features.

\section{An overview of features}

PySPH currently allows a user to set up simulations involving incompressible fluids and free surfaces in two and three dimensions. The framework supports complex geometries. However, only a few simple shapes have been currently implemented. The framework has been designed from the ground up to be parallel. We use mpi4py [mpi4py] for the parallel solver. The parallel solver is automatically load balanced.

In the following, we outline the framework, discuss the current status and future improvements that are planned.

\section{The Framework}

The whole framework was designed to enable simple simulations to be set up very easily, and yet be flexible enough to add complex features. We present a high level view of a particle-based simulation in the following.

\section{Guiding Principle - High level view of a simulation}

A simulation always involves a few key objects:

- Solver: The solver is an object that manages the entire simulation. It typically delegates its activities to other objects like integrators, component managers and arrays of particles.

- Entities: The simulation involves distinct collections of particles each representing a particular physical entity. Each entity is a derived class from the base class EntityBase. For example, Fluid and Solid are two different classes and a user may create a collection of fluids and solids using this. This allows a user to set up a simulation with a collection of physical entities.

The high level view outlined in Figure 1 served as the guiding principle while designing various components of the framework.

The various tasks shown in Figure 1 are explained below:

- Create and set up the solver: Initially, we create an appropriate solver object for the simulation. Different solvers are used for different kinds of simulations. We also set up various parameters of the solver.

- Create physical entities: In this step, we add the physical entities (made of up particles), that will take part in the simulation. Multiple sets of particles could be added, one for each physical entity involved.
Create and Setup a Solver
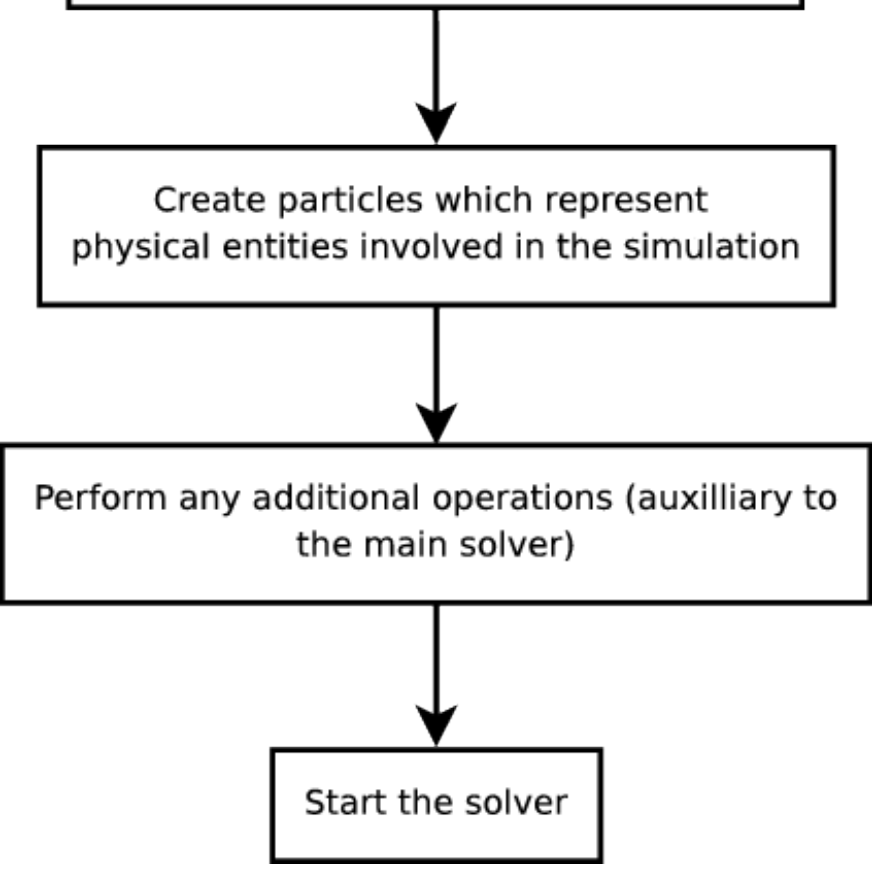

Fig. 1: Outline of tasks to set up a simulation.

- Additional operations to the solver: We may require the solver to perform additional operations (apart from the main simulation), like writing data to file, plotting the data etc. This is configured during this step.

- Start the solver: The solver iterations are started.

The outline given above is very generic. This set of steps is useful in setting up almost any particle-based simulation. Parallel simulations too should adhere to the basic outline given above. Given below is pseudo-Python code to run a simple serial simulation:

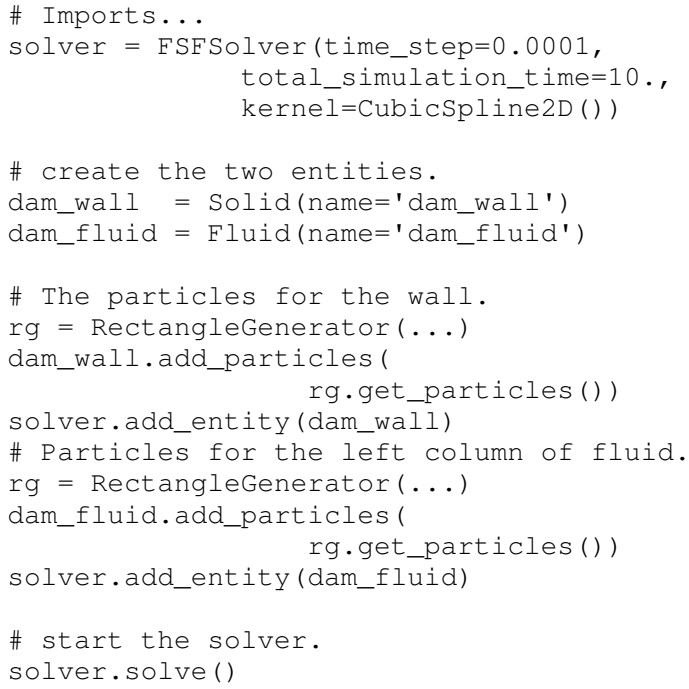




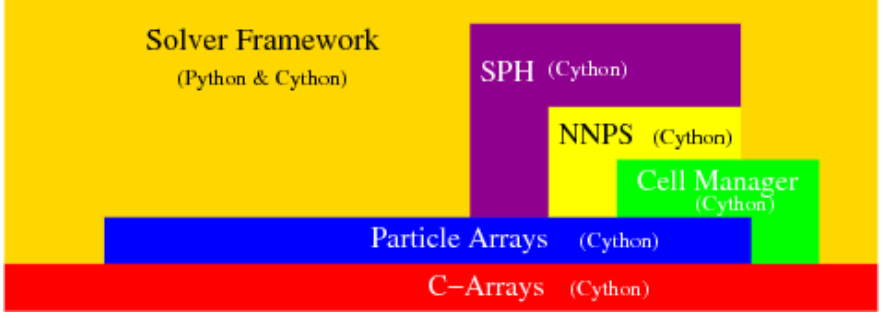

Fig. 2: Architecture of the framework

\section{Architecture Overview}

The architecture may be broadly split into the following:

- the particle kernel,

- the SPH kernel,

- the solver framework,

- $\quad$ serial and parallel solvers.

The overall architecture of the framework is shown in Figure 2. We discuss this in detail in the following sections.

\section{Particle kernel}

A fast implementation of arrays in Cython forms the foundation of the framework. Arrays are ubiquitous in the implementation, hence the implementation is made as fast as possible (close to $\mathrm{C}$ performance) using Cython. The base class for these arrays is called BaseArray and subclasses of these in the form of IntArray, FloatArray etc. are made available. These expose a get_npy_array method which returns a numpy array which internally uses the same $\mathrm{C}$ data buffer. Our arrays may be resized and are up to 4 times faster than numpy arrays when used from Cython.

The ParticleArray module uses these arrays extensively and allows us to represent collections of particles in the framework. It is also implemented in Cython to achieve maximum performance. Each ParticleArray maintains a collection of particle properties and uses the arrays to store this data. Since the arrays allow the developer to manipulate them as numpy arrays, it becomes easy to perform calculations on the particle properties, if required.

One of the central requirements of the $\mathrm{SPH}$ is to find the nearest neighbors of a given particle. This is necessary in order to calculate the influence of each particle on the others. We do this using a nearest neighbor algorithm (Nearest Neighbor Particle Search - NNPS) which bins the domain into a collection of fixed size cells. Particles are organized into a dictionary keyed on a tuple indicative of the location of the particle. The nearest neighbor search is collectively performed by the CellManager class and the nnps modules. Both are implemented in Cython.

\section{SPH kernel}

The SPH kernel consits of the sph module which contains classes to perform the SPH summation (as given in the equations in the introductory section) and also to represent particle interactions. This includes a variety of kernels. These are implemented so as to use the nnps and other modules discussed earlier. These are all implemented in Cython for performance.

\section{Solver framework}

Finally, bringing all the underlying modules together is the Solver framework. The framework is component based, and allows users to write components, which are subclasses of SolverComponent, with a standard interface set. The SolverComponent is the base class for all classes that perform any operation on any of the entities. Many abstractions required for a solver have been implemented, and a user can inherit from various classes to implement new formulations. The ComponentManager manages all the SolverComponents used by the solver. It is also responsible for the property requirements of each of the components involved in a calculation. Thus, if an entity is operated by a component that requires a particular property to be available, the manager ensures that the entity is suitably set up. An Integrator class handles the actual time integration. The Integrator is also a SolverComponent. These are implemented in a combination of Python and Cython.

\section{Solvers}

New solvers are written using the various abstractions developed in the solver framework and all of them derive from the SolverBase class. Serial and parallel solvers are written using the functionality made available in the solver framework.

\section{Parallelization}

In SPH simulations, particles simply influence other particles in a small neighborhood around them. Thus, in order to perform a parallel simulation one needs to:

- partition the particles among different processors, and

- share neighboring particle information between some of the processors.

For an SPH simulation, this does require a reasonable amount of communication overhead since the particles are moving and the neighbor information keeps changing. In addition to this, we would like the load on the processors to be reasonably balanced. This is quite challenging.

Our objective was to maintain an outline similar to the serial code for setting up simulations that run in parallel. For parallelization of the framework, ideally only the CellManager needs to be aware of the parallelism. The components in the solver framework simply operate on particle data that they are presented with. This is achievable to a good extent, except when a component requires global data, in which case the serial component may need to subclassed and a parallel version written, which collects the global data before executing the serial version code. A good example for this is when a component needs to know the maximum speed of sound in the entire domain in order to limit the time-step say.

The pseudo-code of a typical parallel simulation is the same as the serial example given earlier with just one change to the solver as below:

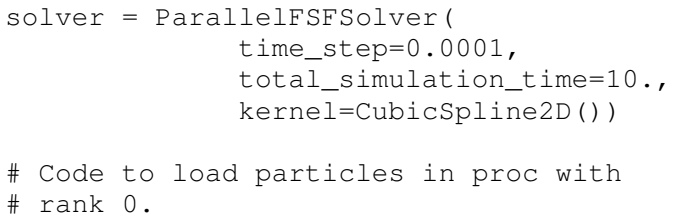

In the above pseudo-code, the only thing that changes is the fact that we instantiate a parallel solver rather than a serial one. 
Serial case

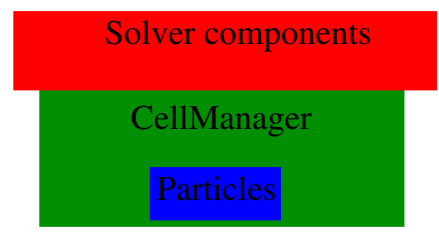

Parallel case

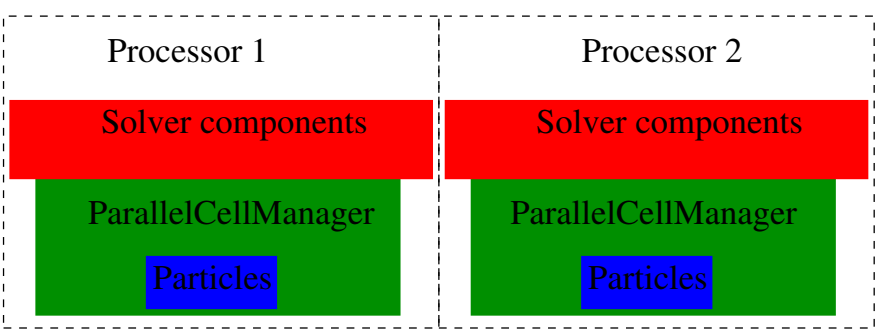

Fig. 3: The parallel solvers simply use a ParallelCellManager instead of a CellManager.

We also ensure that the particles are all loaded only on the first processor. The ParallelCellManager manages the parallel neighbor information. It also performs automatic load-balancing by distributing the particles to different processors on demand based on the number of particles in each processor.

The full details of the parallelization are beyond the scope of this article but we provide a brief outline of the general approach. More details can be obtained from [Kaushik09].

The basic idea of the parallelization involves the following key steps:

- Particles are organized into small cubical Cells. Each cell manages a set of particles. Cells are created and destroyed on demand depending on where the particles are present.

- A region consists of a set of usually (but not always) connected cells. Each region is managed by one processor.

- The domain of particles is decomposed into cells and regions and allocated to different processors.

- Cells are moved between processors in order to balance the load.

In addition, the ParallelCellManager ensures that each processor has all the necessary information such that an SPH computation may be performed on the the particles it manages.

Figure 3 outlines how the parallel and serial solvers are set up internally. In both cases, solver components operate on cell managers to obtain the nearest neighbors and get the particles, the only difference being the ParallelCellManager, which manages the load distribution and communication in the parallel case.

It is important to note that the basic ideas for the parallel algorithm were implemented and tested in pure Python using mpi4py. This was done in highly fragmented time and was possible only because of the convenience of both Python and mpi4py. Mpi4py allows us to send Python objects to processors and this allowed us to focus on the algorithm without worrying about the details of MPI. The use of Python enabled rapid prototyping and its libraries made it easy to visualize the results. In roughly

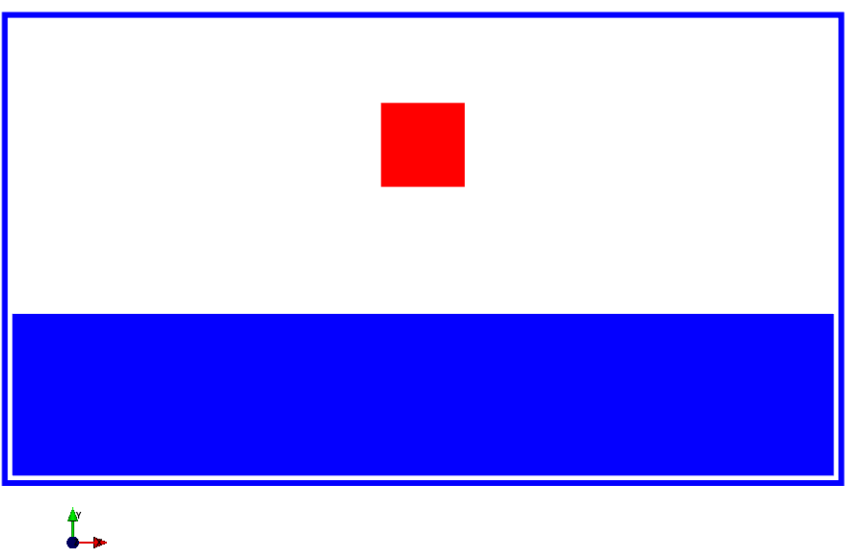

Fig. 4: Initial condition of a square block of water falling towards a vessel with water.

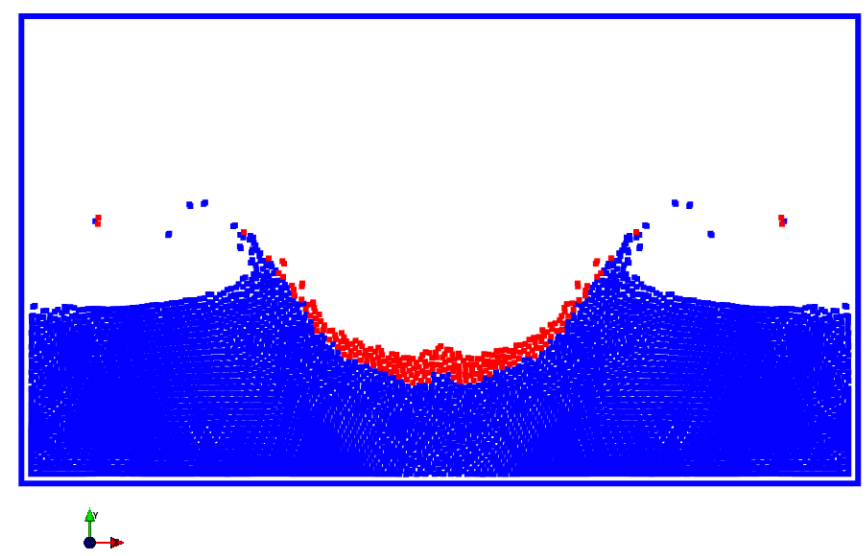

Fig. 5: Square block of water after it strikes a vessel containing water simulated with the SPH.

1500 lines we had implemented the core ideas, added support for visualization, logging and command line options. The initial design was subsequently refined and parts of it implemented in Cython. Thus, the use of Python clearly allowed us to prototype rapidly and yet obtain good performance with Cython.

\section{Current status}

Figures 4,5 show the fluid at a particular instant when a square block of water strikes a vessel filled with water. This is a twodimensional simulation.

Figure 6 shows a typical 3D dam-break problem being simulated with 8 processors. The fluid involved is water. The colors indicate the processor on which the particles are located.

The current capabilities of PySPH include the following:

- Fully automatic, load balanced, parallel framework.

- Fairly easy to script.

- Good performance.

- Relatively easy to extend.

- Solver for incompressible free surface flows. 


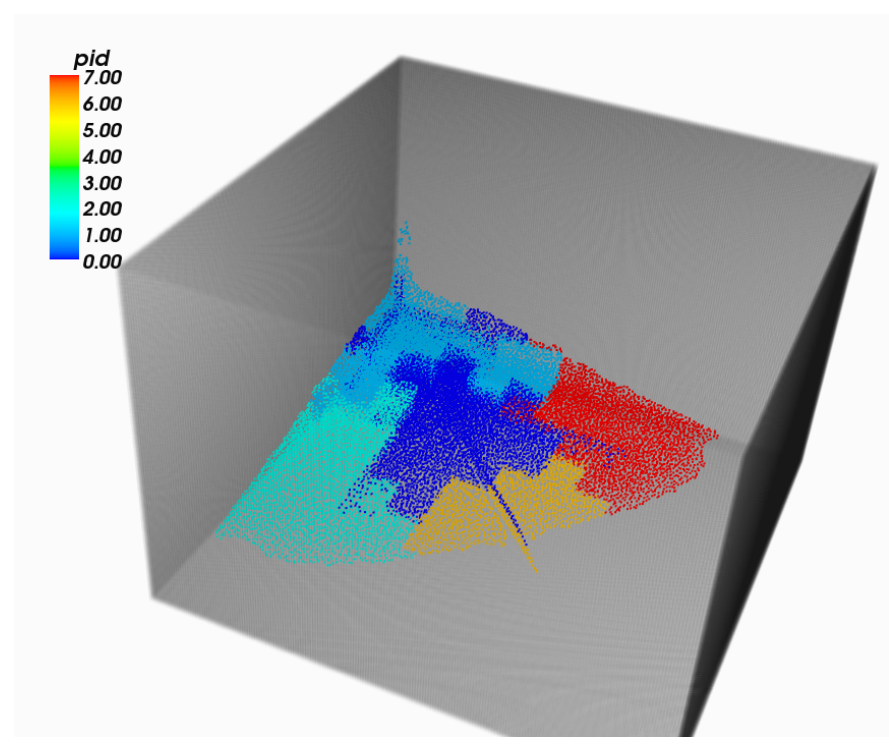

Fig. 6: $3 D$ dam-break problem simulated on 8 processors with particles colored as per processor ID indicating a load balanced simulation.

Most importantly, we have a working framework and a reasonable design which provides good performance. However, there are several things we need to improve.

\section{Future work}

Our code is available in the form of a Mercurial repository on Google's project hosting site [PySPH]. However, the code is not ready for a proper release yet because we would like to perform a redesign of some parts of the solver framework. At the moment, they are a little too complex. Once this is done we would like to do the following:

- Improve the documentation.

- Reduce any compulsory dependence on VTK or TVTK.

- Improve testing on various platforms.

- A full-fledged release.

- Support for gas-dynamics problems.

- Support for solid mechanics problems.

This would take a few more months and at which point we will make a formal release.

\section{Conclusions}

We have provided a high-level description of the current capabilities and architecture of PySPH. We have also mentioned what we believe are the future directions we would like to take. We think we have made an important beginning and believe that PySPH will help enable open research and computing using particle-based computing in the future. It is important to note that Python has been centrally important in the development of PySPH by way of its rapid prototyping capability and access to a plethora of libraries.

\section{REFERENCES}

[Cython] http://www.cython.org
[Gingold77] R. A. Gingold and J. J. Monaghan. Smoothed particle hydrodynamics: theory and application to non-spherical stars, Mon. Not. R. astr. Soc., 181:375-389, 1977.

[Kaushik09] Chandrashekhar P. Kaushik. A Python based parallel framework for Smoothed Particle Hydrodynamics, M.Tech. dissertation, Department of Computer Science and Engineering, IIT Bombay, 2009.

[Lucy77] L. B. Lucy. A numerical approach to testing the fission hypothesis, The Astronomical Journal, 82(12):1013-1024, December 1977

[Monaghan05] J. J. Monaghan. Smoothed particle hydrodynamics, Reports on Progress in Physics, 68(8):1703-1759, 2005.

[Morris96] J. P. Morris. Analysis of smoothed particle hydrodynamics with applications, PhD Thesis, Monash University, Australia, 1996.

[Morris97] J. P. Morris, P. J. Fox and Yi Zhu. Modeling low Reynolds number incompressible flows using $S P H$, Journal of Computational Physics, 136(1):214-226, 1997.

[mpi4py] http://mpi4py.scipy.org

[Oliphant07] Travis E. Oliphant. Python for scientific computing, Computing in science and engineering, 9:10-20, 2007.

[PySPH] http://pysph.googlecode.com

[SPH2000] S. Ganzenmuller, S. Pinkenburg and W. Rosenstiel. SPH2OOO: A Parallel Object-Oriented Framework for Particle Simulations with SPH, Lecture notes in computer science, 3648:1275$1284,2005$.

[SPHysics] Gòmez-Gesteira M., Rogers, B.D., Dalrymple, R.A., Crespo, A.J.C. and Narayanaswamy, M. User guide for the SPHysics code 1.4, http://wiki.manchester.ac.uk/sphysics. 